\title{
ON A CHARACTERIZATION OF GENERALIZED ERLANG-TRUNCATED EXPONENTIAL DISTRIBUTION THROUGH DISTRIBUTIONAL PROPERTIES OF DUAL GENERALIZED ORDER STATISTICS
}

\author{
IMTIYAZ A. SHAH
}

\begin{abstract}
Generalized Erlang-truncated exponential distribtion $F(x)=\left[1-e^{-\beta\left(1-e^{-\lambda}\right) x}\right]$ has been characterized through translation of two non-adjacent dual generalized order statistics (dgos) and then the characterizing results are obtained for generalized Pareto distribution through dilation of dual generalized order statistics (dgos) and generalized power function distribution through contraction of non-adjacent generalized order statistics (gos). Further, the results are deduced for order statistics, lower record statistics, upper record statistics and adjacent generalized order statistics and dual generalized order statistics.
\end{abstract}

\section{Introduction}

Kamps [6] introduced the concept of generalized order statistics ( $g o s$ ) as follows: Let $X_{1}, X_{2}, \ldots, X_{n}$ be a sequence of independent and identically distributed ( $i$ id) random variables $(r v)$ with the absolutely continuous distribution function $(d f) F(x)$ and the probability density function ( $p d f) f(x), x \in(a, b)$. Let $n \in N, n \geq 2, k>0, \tilde{m}=\left(m_{1}, m_{2}, \ldots, m_{n-1}\right) \in \Re^{n-1}$, $M_{r}=\sum_{j=r}^{n-1} m_{j}$, such that $\gamma_{r}=k+(n-r)+M_{r}>0$ for all $r \in\{1,2, \ldots, n-1\}$. If $m_{1}=m_{2}=\cdots=$ $m_{n-1}=m$, then $X(r, n, m, k)$ is called the $r^{\text {th }} m-g o s$ and its $p d f$ is given as:

$$
f_{X(r, n, m, k)}(x)=\frac{C_{r-1}^{(n)}}{(r-1) !}[\bar{F}(x)]^{\gamma_{i}^{(n)}-1} g_{m}^{r-1}(F(x)) f(x), \quad a<x<b
$$

where

$$
C_{r-1}^{(n)}=\Pi_{i-1}^{r} \gamma_{i}^{(n)}, \quad \gamma_{i}^{(n)}=k+(n-i)(m+1), \quad 1 \leq r \leq n
$$

and

$$
g_{m}(x)=h_{m}(x)-h_{m}(0), x \in[0,1) .
$$

Received August 26, 2012, accepted January 30, 2013.

Communicated by Ming-Yen Cheng. 


$$
h_{m}(x)= \begin{cases}-\frac{1}{m+1}(1-x)^{m+1} & , m \neq-1 \\ -\log (1-x) & , m=-1\end{cases}
$$

Now since $\lim _{m \rightarrow-1} h_{m}(x)=-\lim _{m \rightarrow-1} \frac{(1-x)^{m+1}}{m+1}=-\log (1-x)$, therefore, we will consider $h_{m}(x)$ $=-\frac{1}{m+1}(1-x)^{m+1}$, for all $m$, unless otherwise needed.

Based on the generalized order statistics (gos), Burkschat et al. [4] introduced the concept of the dual generalized order statistics (dgos) where the $p d f$ of the $r^{\text {th }} m-d g o s$ $X^{*}(r, n, m, k)$ is given as

$$
f_{X^{*}(r, n, m, k)}(x)=\frac{C_{r-1}^{(n)}}{(r-1) !}[F(x)]^{\gamma_{i}^{(n)}-1} g_{m}^{r-1}(F(x)) f(x), \quad a<x<b
$$

which is obtained just by replacing $\bar{F}(x)=1-F(x)$ by $F(x)$

where

$$
h_{m}(x)= \begin{cases}-\frac{1}{m+1} x^{m+1} & , m \neq-1 \\ -\log x & , m=-1\end{cases}
$$

and

$$
g_{m}(x)=h_{m}(x)-h_{m}(1), x \in[0,1) .
$$

since $-\lim _{m \rightarrow-1} \frac{1}{m+1} x^{m+1} \rightarrow-\log x$, therefore, we will consider $h_{m}(x)=-\frac{1}{m+1} x^{m+1}$, for all $m$, unless otherwise needed.

If support of the distribution $F(x)$ be over $(a, b)$, then by convention, we will write

$$
X(0, n, m, k)=a \quad \text { and } \quad X^{*}(0, n, m, k)=b
$$

Ahsanullah [1] has characterized uniform distribution under random contraction for adjacent $d g o s$. Khan and Shah [7] have characterized distributions using distributional properties of non- adjacent lower records, upper records and order statistics. In this paper, distributional properties of the $d g o s$ have been used to characterize a general form of Erlangtruncated exponential distribution for non- adjacent $d g o s$ under random translation, dilation and contraction, thus generalizing the results of Ahsanullah [1]. Further, results in terms of lower records, upper records and order statistics are deduced. One may also refer to Alzaid and Ahsanullah [2], Wesolowski and Ahsanullah [8], Beutner and Kamps [3] and CastañoMartínez et al. [5] for the related results.

It may be seen that if $Y$ is a measurable function of $X$ with the relation

$$
Y=h(X)
$$


then

(i) $Y^{*}(r, n, m, k)=h\left[X^{*}(r, n, m, k)\right]$

(ii) $Y_{r: n}=h\left(X_{r: n}\right)$

(iii) $Y_{L(r)}=h\left(X_{L(r)}\right)$

if $h$ is an increasing function and

(i) $Y(r, n, m, k)=h\left[X^{*}(r, n, m, k)\right]$

(ii) $Y_{n-r+1: n}=h\left(X_{r: n}\right)$

(iii) $X_{U(r)}=h\left(X_{L(r)}\right)$

if $h$ is a decreasing function

where $X_{r: n}$ is the $r^{\text {th }}$ order statistics from a sample of size $n, X_{U(r)}$ is the $r^{\text {th }}$ upper record and $X_{L(r)}$ is the $r^{\text {th }}$ lower record, $X(r, n, m, k)$ is the $r^{\text {th }} m-g o s$ and $X^{*}(r, n, m, k)$ is the $r^{\text {th }}$ $m-d g o s$.

We will denote

(i) $X \sim$ Erlang-truncated $\exp \left(\beta\left(\alpha_{\lambda}\right)\right)$

if $X$ has an Erlang-truncated exponential distribution with the $d f$

$$
F(x)=\left[1-e^{-\beta\left(\alpha_{\lambda}\right) x}\right], \quad 0 \leq x<\infty, \quad \beta>0, \lambda>0
$$

where $\alpha_{\lambda}=\left(1-e^{-\lambda}\right)$

(ii) $X \sim \operatorname{Par}\left(\beta\left(\alpha_{\lambda}\right)\right)$

if $X$ has a Pareto distribution with the $d f$

$$
F(x)=\left[1-x^{-\beta\left(\alpha_{\lambda}\right)}\right], \quad 1<x<\infty, \quad \beta>0, \lambda>0
$$

(iii) $X \sim \operatorname{pow}\left(\beta\left(\alpha_{\lambda}\right)\right)$

if $X$ has a power function distribution with the $d f$

$$
F(x)=x^{\beta\left(\alpha_{\lambda}\right)}, \quad 0<x<1, \quad \beta>0, \lambda>0
$$

(iv) $X \sim$ genErlang-truncated $\exp \left(\beta\left(\alpha_{\lambda}\right)\right)$

if $X$ has a generalized Erlang-truncated exponential distribution with the $d f$

$$
F(x)=\left[1-(m+1) e^{-\beta\left(\alpha_{\lambda}\right) x}\right]^{\frac{1}{m+1}}, \quad \frac{1}{\beta\left(\alpha_{\lambda}\right)} \ln (m+1)<x<\infty, \quad \beta>0
$$

(v) $X \sim \operatorname{genPar}\left(\beta\left(\alpha_{\lambda}\right)\right)$ 
if $X$ has a generalized Pareto distribution with the $d f$

$$
F(x)=\left[1-(m+1) x^{-\beta\left(\alpha_{\lambda}\right)}\right]^{\frac{1}{m+1}}, \quad(m+1)^{\frac{1}{\beta\left(\alpha_{\lambda}\right)}}<x<\infty, \quad \beta>0
$$

(iii) $X \sim \operatorname{genpow}\left(\beta\left(\alpha_{\lambda}\right)\right)$

if $X$ has a generalized power function distribution with the $d f$

$$
F(x)=1-\left[1-(m+1) x^{\beta\left(\alpha_{\lambda}\right)}\right]^{\frac{1}{m+1}}, \quad 0<x<(m+1)^{\frac{1}{\beta\left(\alpha_{\lambda}\right)}}, \beta>0
$$

It may further be noted that

$$
\begin{aligned}
\text { if } \quad \log X \sim \text { Erlang-truncated } \exp \left(\beta\left(\alpha_{\lambda}\right)\right) \text { then } X \sim \operatorname{Par}\left(\beta\left(\alpha_{\lambda}\right)\right) \\
\text { if }-\log X \sim \text { Erlang-truncated } \exp \left(\beta\left(\alpha_{\lambda}\right)\right) \text { then } X \sim \operatorname{pow}\left(\beta\left(\alpha_{\lambda}\right)\right) \\
\text { if } \quad \log X \sim \text { genErlang-truncated } \exp \left(\beta\left(\alpha_{\lambda}\right)\right) \text { then } X \sim \operatorname{genPar}\left(\beta\left(\alpha_{\lambda}\right)\right) \\
\text { and if }-\log X \sim \text { genErlang-truncated } \exp \left(\beta\left(\alpha_{\lambda}\right)\right) \text { then } X \sim \operatorname{genpow}\left(\beta\left(\alpha_{\lambda}\right)\right)
\end{aligned}
$$

It has been assumed here throughout that the $d f$ is differentiable w.r.t. its argument.

\section{Characterizing results}

Theorem 2.1. Let $X^{*}(s, n, m, k)$ be the $s^{\text {th }} m$-dgos from a sample of size $n$ drawn from $a$ continuous population with the pd $f(x)$ and the $f f F(x)$, then

$$
X^{*}(s-j, n, m, k) \stackrel{d}{=} X^{*}(s, n, m, k)+Y_{j: s-1}, j=s-r-1, s-r ; 1 \leq r<s \leq n
$$

where $Y_{j: s-1}$ is the $j^{\text {th }}$ order statistic from a sample of size $(s-1)$ drawn from Erlang-truncated $\exp \left(\beta\left(\alpha_{\lambda}\right)\right.$ distribution and is independent of $X^{*}(s, n, m, k)$ if and only if $X_{1} \sim$ genErlang-truncated $\exp \left(\beta\left(\alpha_{\lambda}\right)\right)$ and $X \stackrel{d}{=} Y$ denotes that $X$ and $Y$ have the same $d f$

Proof. To prove the necessary part, let the moment generating function $(m g f)$ of $X^{*}(r, n, m, k)$ be $M_{X_{(r)}^{*}}(t)$, then

$$
X^{*}(r, n, m, k) \stackrel{d}{=} X^{*}(s, n, m, k)+Y
$$

which implies that

$$
M_{X_{(r)}^{*}}(t) \stackrel{d}{=} M_{X_{(s)}^{*}}(t) \cdot M_{Y}(t)
$$

Since for the genErlang-truncated $\exp \left(\beta\left(\alpha_{\lambda}\right)\right)$ distribution.

$$
M_{X_{(r)}^{*}}(t)=\frac{C_{r-1}^{(n)}}{(r-1) !} \frac{1}{(m+1)^{r-\frac{t}{\beta\left(\alpha_{\lambda}\right)}}} \frac{\Gamma\left(r-\frac{t}{\beta\left(\alpha_{\lambda}\right)}\right) \Gamma\left(\frac{\gamma_{r}^{(n)}}{(m+1)}\right)}{\Gamma\left(r-\frac{t}{\beta\left(\alpha_{\lambda}\right)}+\frac{\gamma_{r}^{(n)}}{m+1}\right)}
$$


Therefore

$$
M_{Y}(t)=\frac{M_{X_{(r)}^{*}}(t)}{M_{X_{(s)}^{*}}(t)}=\frac{\Gamma(s) \Gamma\left(r-\frac{t}{\beta\left(\alpha_{\lambda}\right)}\right)}{\Gamma(r) \Gamma\left(s-\frac{t}{\beta\left(\alpha_{\lambda}\right)}\right)}
$$

But this is the $\operatorname{mg} f$ of $Y_{s-r: s-1}$, the $(s-r)^{\text {th }}$ order statistic from a sample of size $(s-1)$ drawn from Erlang-truncated $\exp \left(\beta\left(\alpha_{\lambda}\right)\right.$ and hence the result.

For the proof of sufficiency part, we have by the convolution method

$$
\begin{aligned}
f_{X^{*}(r, n, m, k)}(x) & =\int_{0}^{x} f_{X^{*}(s, n, m, k)}(y) f_{Y_{s-r: s-1}}(x-y) d y \\
& =\frac{\beta\left(\alpha_{\lambda}\right)(s-1) !}{(r-1) !(s-r-1) !} \int_{0}^{x}\left[e^{-\beta\left(\alpha_{\lambda}\right)(x-y)}\right]^{r}\left[1-e^{-\beta\left(\alpha_{\lambda}\right)(x-y)}\right]^{s-r-1} f_{X^{*}(s, n, m, k)}(y) d y
\end{aligned}
$$

Differentiate both the sides of (2.2) w.r.t. $x$, to get

$$
\begin{aligned}
& \frac{d}{d x} f_{X^{*}(r, n, m, k)}(x) \\
&= \frac{\beta\left(\alpha_{\lambda}\right)(s-1) !}{(r-1) !(s-r-2) !} \int_{0}^{x} \beta\left(\alpha_{\lambda}\right)\left[e^{-\beta\left(\alpha_{\lambda}\right)(x-y)}\right]^{r+1}\left[1-e^{-\beta\left(\alpha_{\lambda}\right)(x-y)}\right]^{s-r-2} f_{X^{*}(s, n, m, k)}(y) d y \\
&-\frac{\beta\left(\alpha_{\lambda}\right)(s-1) ! r}{(r-1) !(s-r-1) !} \int_{0}^{x} \beta\left(\alpha_{\lambda}\right)\left[e^{-\beta\left(\alpha_{\lambda}\right)(x-y)}\right]^{r}\left[1-e^{-\beta\left(\alpha_{\lambda}\right)(x-y)}\right]^{s-r-1} f_{X^{*}(s, n, m, k)}(y) d y \\
&= \beta\left(\alpha_{\lambda}\right) r\left[f_{X^{*}(r+1, n, m, k)}(x)-f_{X^{*}(r, n, m, k)}(x)\right] \\
& \text { or, } \quad f_{X^{*}(r, n, m, k)}(x)=\beta\left(\alpha_{\lambda}\right) r\left[F_{X^{*}(r+1, n, m, k)}(x)-F_{X^{*}(r, n, m, k)}(x)\right]
\end{aligned}
$$

Now, since (Ahsanullah [1])

$$
F_{X^{*}(r+1, n, m, k)}(x)-F_{X^{*}(r, n, m, k)}(x)=\frac{F(x)}{\gamma_{r+1} f(x)} f_{X^{*}(r+1, n, m, k)}(x)
$$

Therefore, in view of (1.4), (2.3) and (2.4), we have

$$
\frac{(m+1)[F(x)]^{m} f(x)}{\left[1-(F(x))^{m+1}\right]}=\beta\left(\alpha_{\lambda}\right)
$$

implying that

$$
F(x)=\left[1-(m+1) e^{-\beta\left(\alpha_{\lambda}\right) x}\right]^{\frac{1}{m+1}}
$$

and hence the proof.

Remark 2.1. Let $X_{r: n}$ be the $r^{\text {th }}$ order statistic from a sample of size $n$ drawn from a continuous population with the $p d f f(x)$ and the $d f F(x)$, then

$$
X_{r+j: n} \stackrel{d}{=} X_{r: n}+Y_{j: n-r}, j=s-r-1, s-r ; 1 \leq r<s \leq n
$$

where $Y_{j: n-r} \stackrel{d}{=} X_{j: n-r}$ is independent of $X_{r: n}$ if and only if $X_{1} \sim \exp (\beta)$. 
This can be established by noting that order statistic appear in the generalized order statistics ( $g o s$ ) model as well as in dual generalized order statistics ( $d g o s)$ model, therefore at $m=0$ and $\alpha_{\lambda}=\alpha$, (2.1) may be written as

$$
X_{n-s+j+1: n} \stackrel{d}{=} X_{n-s+1: n}+Y_{j: s-1}, j=s-r-1, s-r ; 1 \leq r<s \leq n
$$

which implies

$$
X_{r+j: n} \stackrel{d}{=} X_{r: n}+Y_{j: n-r}, j=s-r-1, s-r ; 1 \leq r<s \leq n
$$

obtained by replacing $(n-s+1)$ by $r$ and $(n-r+1)$ by $s$, as given by Khan and Shah [7].

Remark 2.2. For $\alpha_{\lambda}=\alpha$, Alzaid and Ahsanullah [2] have proved that

$$
X_{r: n} \stackrel{d}{=} X_{r-1: n}+V
$$

where $V \sim \exp (n-r+1)$ if and only if $X_{1} \sim \exp (1)$.

Remark 2.3. For $\alpha_{\lambda}=\alpha$, Castaño-Martínez et al. [5] have shown that

$$
X_{s: n} \stackrel{d}{=} X_{r: n}+V
$$

where $V \stackrel{d}{=}-\log W$ with $W \sim \operatorname{Be}(n-r+1, s-r)$ if and only if $X_{1} \sim \exp (1)$.

Remark 2.4. As $m \rightarrow-1$ and $\alpha_{\lambda}=\alpha$, $\operatorname{genexp}(\beta)$ tends to the $\operatorname{Gum}(\beta)$ and $X^{*}(r, n, m, k)$ to $X_{L(r)}$, the $r^{\text {th }}$ lower records. Therefore we have

$$
X_{L(s-j)} \stackrel{d}{=} X_{L(s)}+Y_{j: s-1}, j=s-r-1, s-r ; 1 \leq r<s \leq n
$$

where $Y_{j: s-1}$ is the $j^{\text {th }}$ order statistic from a sample of size $(s-1)$ drawn from $\exp (\beta)$ distribution and is independent of $X_{L(s)}$ if and only if $X_{1} \sim \operatorname{Gum}(\beta)$, as obtained by Khan and Shah [7].

Remark 2.5. For $\alpha_{\lambda}=\alpha$, Alzaid and Ahsanullah [2] have shown that

$$
X_{L(r)} \stackrel{d}{=} X_{L(r+1)}+V
$$

where $V \sim \exp (r)$ if and only if $X_{1} \sim$ Gum (1).

Corollary 2.2. Let $X^{*}(s, n, m, k)$ be the $s^{\text {th }} m$-dgos from a sample of size $n$ drawn from a continuous population with the pd $f(x)$ and the $f f F(x)$, then

$$
X^{*}(s-j, n, m, k) \stackrel{d}{=} X^{*}(s, n, m, k) \cdot Y_{j: s-1}, j=s-r-1, s-r ; 1 \leq r<s \leq n
$$

where $Y_{j: s-1}$ is the $j^{\text {th }}$ order statistic from a sample of size $(s-1)$ drawn from $\operatorname{Par}\left(\beta\left(\alpha_{\lambda}\right)\right.$ distribution and is independent of $X^{*}(s, n, m, k)$ if and only if $X_{1} \sim \operatorname{genPar}\left(\beta\left(\alpha_{\lambda}\right)\right)$. 
Proof. Here the product $X^{*}(s, n, m, k) \cdot Y_{j: s-1}$ in (2.7) is called random dilation of $X^{*}(s, n, m, k)$ (Beutner and Kamps [3]). Note that if

$$
\log X^{*}(r, n, m, k) \stackrel{d}{=} \log X^{*}(s, n, m, k)+\log Y_{s-r: s-1}
$$

then

$$
X^{*}(r, n, m, k) \stackrel{d}{=} X^{*}(s, n, m, k) \cdot Y_{s-r: s-1}
$$

in view of (1.6), (1.7), (1.18) and (1.20) and the result follows.

Remark 2.6. In case of ordinary order statistics, i.e., at $m=0$ and $\alpha_{\lambda}=\alpha$, we have

$$
X_{r+j: n} \stackrel{d}{=} X_{r: n}+Y_{j: n-r}, j=s-r-1, s-r ; 1 \leq r<s \leq n
$$

where $Y_{j: n-r} \stackrel{d}{=} X_{j: n-r}$ is independent of $X_{r: n}$ if and only if $X_{1} \sim \operatorname{Par}(\beta)$, as obtained by Castaño-Martínez et al. [5] and Khan and Shah [7].

Remark 2.7. As $m \rightarrow-1$ and $\alpha_{\lambda}=\alpha$, we get

$$
X_{L(s-j)} \stackrel{d}{=} X_{L(s)} \cdot Y_{j: s-1}, j=s-r-1, s-r ; 1 \leq r<s \leq n
$$

where $Y_{j: s-1}$ is the $j^{\text {th }}$ order statistic from a sample of size $(s-1)$ drawn from $\operatorname{Par}(\beta)$ distribution and is independent of $X_{L(s)}$, be the $s^{\text {th }}$ lower records if and only if $X_{1} \sim \operatorname{inW}(\beta)$.

Corollary 2.3. Let $X(s, n, m, k)$ be the $s^{\text {th }} m$-gos from a sample of size $n$ drawn from a continuous population with the $p d f f(x)$ and the $d f F(x)$, then

$$
X(s-j, n, m, k) \stackrel{d}{=} X(s, n, m, k) \cdot Y_{s-j: s-1}, j=s-r-1, s-r ; 1 \leq r<s \leq n
$$

where $Y_{s-j: s-1}$ is the $(s-j)^{\text {th }}$ order statistic from a sample of size $(s-1)$ drawn from pow $\left(\beta\left(\alpha_{\lambda}\right)\right.$ distribution and is independent of $X(s, n, m, k)$ if and only if $X_{1} \sim \operatorname{genpow}\left(\beta\left(\alpha_{\lambda}\right)\right)$.

Proof. Here the product $X(s, n, m, k) \cdot Y_{s-j: s-1}$ in (2.8) is called random contraction of $X(s-$ $j, n, m, k)$ (Beutner and Kamps [3]). Since

$$
-\log X^{*}(r, n, m, k) \stackrel{d}{=}-\log X^{*}(s, n, m, k)-\log Y_{s-r: s-1}
$$

implies

$$
X(r, n, m, k) \stackrel{d}{=} X(s, n, m, k) \cdot Y_{r: s-1}
$$

in view of (1.9), (1.10), (1.19) and (1.21) and the result follows.

Remark 2.8. For $\alpha_{\lambda}=\alpha$, Beutner and Kamps [3] have shown that for adjacent generalized order statistics

$$
X(r, n, m, k) \stackrel{d}{=} X(r+1, n, m, k) \cdot V
$$

where $V \sim \operatorname{pow}(\beta)$ if and only if $X_{1} \sim \operatorname{genpow}(\beta)$. 
Remark 2.9. We can get the corresponding characterizing results for the order statistics at $m=0$ and $\alpha_{\lambda}=\alpha$ as:

Let $X_{r: n}$ be the $r^{\text {th }}$ order statistic from a sample of size $n$ drawn from a continuous population with the $p d f f(x)$ and the $d f F(x)$, then

$$
X_{s-j: n} \stackrel{d}{=} X_{s: n} \cdot Y_{s-j: s-1}, j=s-r-1, s-r ; 1 \leq r<s \leq n
$$

where $Y_{s-j: s-1} \stackrel{d}{=} X_{s-j: s-1}$ is independent of $X_{s: n}$ if and only if $X_{1} \sim \operatorname{pow}(\beta)$, as given by Khan and Shah [7].

For adjacent order statistics one may also refer to Ahsanullah [1], Wesolowski and Ahsanullah [8].

Remark 2.10. The corresponding result for the lower records as $m \rightarrow-1$ and $\alpha_{\lambda}=\alpha$ is:

Let $X_{U(s)}$ be the $s^{\text {th }}$ upper record from a continuous population with the $\operatorname{pd} f(x)$ and the $d f F(x)$, then

$$
X_{U(s-j)} \stackrel{d}{=} X_{U(s)} \cdot Y_{s-j: s-1}, j=s-r-1, s-r ; 1 \leq r<s
$$

where $Y_{s-j: s-1}$ is the $(s-j)^{\text {th }}$ order statistic from a sample of size $(s-1)$ drawn from pow $(\beta)$ distribution and is of $X_{U(s)}$ if and only if $X_{1} \sim W e i(\beta)$, as obtained by Khan and Shah [7].

\section{Acknowledgements}

The authors are thankful to the Referee and Editor of Tamkang Journal Mathematics for their fruitful suggestions.

\section{References}

[1] M. Ahsanullah, A characterization of the uniform distribution by dual generalized order statistics, Communications in Statistics - Theory and Methods, 33(2004), 2921-2928.

[2] A. A. Alzaid and M. Ahsanullah, A characterization of the Gumbel distribution based on record values, Commun. Statist. Theor. Meth., 32(2003), 2101-2108.

[3] M. Burkschat, E. Cramer and U. Kamps, Dual generalized order statistics, Metron, LXI(I)(2003), $13-26$.

[4] E. Beutner and U. Kamps, Random contraction and random dilation of generalized order statistics, Commun. Statist. Theor. Meth., 37, 2185-2201.

[5] A. Castaño-Martínez, F. López-Blázquez and B. Salamanca-Miño, Random translations, contractions and dilations of order statistics and records, Statist. (2010), 1-11. DOI: 10.1080/02331888.2010.495406.

[6] U. Kamps, A Concept of Generalized Order Statistics, B. G. Teubner, Stuttgart, 1995.

[7] A. H. Khan and Imtiyaz A. Shah, Distributional properties of order statistics and record statistics, Pak. J. Statist. Oper. Res., 8(3)(2012).

[8] S. Y. Oncel, M. Ahsanullah, F. A. Aliev and F. Aygun, Switching record and order statistics via random contraction, Statist. Probab. Lett., 73 (2005), 207-217.

[9] J. Wesolowski and M. Ahsanullah, Switching order statistics through random power contractions, Aust. N. Z. J. Statist., 46 (2004), 297-303.

Department of Statistics and Operations Research, Aligarh Muslim University, Aligarh-202 022, India.

E-mail: masoom.immy03@gmail.com 